\title{
ESTRUTURA POPULACIONAL DO ARATU Goniopsis cruentata (LATREILLE, 1803) NO ESTUÁRIO DOS RIOS PIAUI/REAL, ESTADO DE SERGIPE, BRASIL
}

\section{POPULATION STRUCTURE OF ARATU Goniopsis cruentata (LATREILLE, 1803) IN THE STUARY OF PIAUI/REAL RIVERS, SERGIPE STATE, BRAZIL}

\author{
Ana Rosa da Rocha Araújo ${ }^{1}$, Paulo Ricardo Ramos Santos ${ }^{2}$, Inajara Marques Bezerra Oliveira ${ }^{3}$,

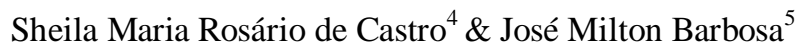 \\ Departamento de Engenharia de Pesca e Aquicultura, Universidade Federal de Sergipe - UFS \\ E-mail: anarosaaraujop@gmail.com
}

Recebido em 20/12/2020 - Publicado em 26/03/2021

RESUMO A pescaria artesanal de aratu ocorre em toda a costa do nordeste, mas a sua captura total só é estimada para três estados, Pernambuco, Sergipe e Bahia. A pesca de aratu é intensa e poucos estudos sobre a biologia da espécie, dinâmica de população e relação socioeconômica tem sido publicado. Dentro desse contexto, esse trabalho teve como objetivo principal caracterizar a estrutura populacional e aspectos reprodutivos do G. cruentata, desembarcado nas comunidades de Cajazeiras e Pontal, Sergipe. As coletas foram efetuadas mensalmente na lua nova, no período de março de 2015 a fevereiro de 2016. Os resultados mostraram que a largura de carapaça (LC) dos animais variou no em Cajazeiras de 25 a 53,8 mm e no Pontal de 25 a 59,6 mm. Cajazeiras e Pontal apresentaram padrões diferentes para a porcentagem de fêmeas e machos, considerando a frequência de largura de carapaça. As fêmeas foram predominantes nas classes de 26 a $48 \mathrm{~mm}$ em Cajazeiras e no Pontal de 28 a $44 \mathrm{~mm}$. Os machos foram maioria nos maiores comprimentos em Cajazeiras e Pontal, o resultado indica que os machos dominam nos maiores comprimentos. Fêmeas maduras foram encontradas durante todo o estudo com maiores frequência no mês de setembro nos dois manguezais estudados. O resultado estimado da largura de carapaça média na primeira maturação de fêmeas em Cajazeiras foi de $33,2 \mathrm{~mm}$ para fêmeas e machos $36,5 \mathrm{~mm}$; e no Pontal de $31,2 \mathrm{~mm}$ para fêmeas e $36,8 \mathrm{~mm}$ para machos.

Palavras-chave: pescadoras, pesca artesanal, reprodução.

\begin{abstract}
Artisanal fishing of aratu occurs throughout the northeastern coast, but their total catch is only estimated for three states, Pernambuco, Sergipe and Bahia. The aratu fishing is intense and few studies on the biology of the species, population dynamics and socioeconomic has been published. Therefore, this work aimed to characterize the population structure and reproductive aspects of $G$. cruentata, landed in the communities of Cajazeiras and Pontal, in Sergipe. The collections were made monthly when the new moon, from March 2015 to February 2016. The results showed that the carapace width (LC) of the animals ranged in Cajazeiras 25 to $53.8 \mathrm{~mm}$ and $59.6 \mathrm{~mm}$ in Pontal. Cajazeiras and Pontal showed different patterns for the percentage of females and males, considering the frequency of carapace width. Females were predominant in 26-48 $\mathrm{mm}$ class in Cajazeiras and in Pontal from 28 to 44 $\mathrm{mm}$. The males were the majority in the longest lengths in Cajazeiras and Pontal, the result indicates that the males dominate in the longest lengths. Mature female maturation was found throughout the study with greater frequency in September in both mangroves studied. The estimated result of the wide average carapace at first maturity of females in Cajazeiras indicated value of $33.2 \mathrm{~mm}$ and males equal to $36.5 \mathrm{~mm}$; Pontal for the female values was $31.2 \mathrm{~mm}$ and $36.8 \mathrm{~mm}$ male.
\end{abstract}

Key words: fisherwomen, artisanal fishing, reproduction. 


\section{Introdução}

Os crustáceos são considerados pelas comunidades locais um dos recursos mais importantes da fauna dos manguezais (Melo, 1996). A ordem dos decápodes constitue um dos maiores grupos entre os crustáceos, com aproximadamente 10.000 espécies, das quais cerca de $89 \%$ vivem em ambientes de influência marinha (Bowman \& Abele, 1982). Dentro do grupo, a infra-ordem Brachyura, na qual os aratus fazem parte, representa um grupo de grande interesse biológico, econômico e social, além de se destacar como fundamental na dinâmica das relações estabelecidas nos mais diversos ecossistemas do litoral brasileiro (Moura \& Coelho, 2004; Maciel \& Alves, 2009).

A família Grapsidae possui quatorze gêneros, e o gênero Goniopsis (Haan, 1833) possui três espécies: $G$. pelli Herklots 1851, G. cruentata e G. pulchra Lockingting, 1876 (Sternberg, 1994). No Brasil foi registrado somente a ocorrência da espécie G. cruentata, o qual ocorre no Atlântico Ocidental - Bermudas, Flórida, México, Antilhas, Guianas e Brasil (do Pará até Santa Catarina, além do arquipélago de Fernando de Noronha e o Atol das Rocas). Ocorre também no Atlântico Oriental - do Senegal a Angola (Melo, 1996; Santos et. al., 2013).

$\mathrm{O}$ aratu é um decápode semiterrestre, pertencente ao subfilo Crustacea, bastante ágil, capaz de deslocarse rapidamente entre as raízes das árvores do manguezal, onívoro, ocorre em manguezais, praias lodosas, em braços de mar ou estuários, do supra litoral até entre marés (Coelho, 1965; Melo,1996; Oshiko et al., 1998; Sousa \& Calado, 2000; Santos et al 2001; Botelho et al, 2004). Conhecido, popularmente no Brasil, de acordo com a região do país, como aratu, maria-mulata, aratu-do-mangue, aratu-vermelho, aratuvermelho-preto, anajá ou bonitinho.

$\mathrm{O}$ aratu ocorre em todos os manguezais no Brasil. No Nordeste, as capturas ocorrem em toda a costa, com maior intensidade nos estados de Pernambuco, Sergipe e Bahia (Santos et al., 2013). Esta espécie se destaca pelo seu valor econômico, constituindo importante fonte de renda para as populações pesqueiras locais (Moura \& Coelho, 2004).

A pesca de aratu é intensa e poucos estudos sobre a biologia da espécie, dinâmica de população e importância socioeconômica tem sido publicado. Estudos sobre essa espécie ainda são escassos, destacando-se, aqueles realizados por: Fransozo et al. (1998) que descreveram o estágio larval zoea I; Cobo e Fransozo (1998, 1999), que estudaram sobre os aspectos de reprodução e crescimento. Aspectos da estrutura populacional como a razão sexual e a estrutura etária, influenciam a dinâmica populacional devido às variações relacionadas ao sexo e à idade no nascimento e na morte, sendo tais aspectos de suma importância para a compreensão da ocorrência de mudanças populacionais ao longo do tempo (Ricklefs, 2010).

O estudo das constantes mudanças nas populações, causadas pelas taxas de migrações, natalidade/mortalidade, e interações bióticas e abióticas podem colaborar para um entendimento da dinâmica populacional e ajudar a esclarecer a estrutura da comunidade e a função ecossistêmica (Ricklefs, 2004). Um aspecto importante de uma estrutura de população é a sua distribuição de tamanho, indicando as proporções de seus membros ao longo de cada classe de idade (Pianka, 1974). Para os crustáceos, a caracterização da estrutura populacional tem sido feita com base na análise dessas frequências dos membros da população, que constituem o número de observações que ocorrem em cada classe de tamanho (Poole, 1974). Essas informações são utilizadas para estimar a intensidade e o período de recrutamento juvenil nas populações, as quais podem explicar variações sazonais do tamanho das populações (Leme, 1999). Para estudos de dinâmica populacional, a razão sexual é estimada comparando a proporção de machos e fêmeas em amostras obtidas a partir de uma determinada população (Haley, 1979).

De acordo com Moura \& Coelho (2004), o tamanho de maturidade sexual em braquiúros tem sido determinado de várias formas, sendo baseados em análises de maturidade morfológica, critérios de crescimento relativo; maturidade fisiológica, a partir de observações sobre a maturação gonadal e maturidade funcional, em observações comportamentais da corte e cópula (Hartnoll, 1985). A proporção entre machos e fêmeas é uma informação importante para a caracterização da estrutura de uma espécie ou população, além de fornecer subsídios para estudo de aspectos como avaliação do potencial reprodutivo e em estimativas do tamanho do estoque (Vazzoler, 1996).

Em Sergipe a pesca do aratu teve uma produção de cerca de 149 toneladas em 2014 (Araújo et al, 2016). Os municípios que apresentaram maior volume de captura foi Santa Luzia do Itanhy e Indiaroba. As capturas de aratu ocorrem durante o dia e a noite. Segundo relatos das pescadoras as capturas de aratu durante a noite costumam ser mais produtiva, pois, a utilização de fonte luminosa serve para iluminar o 
mangue e auxiliam na captura. O feixe de luz é direcionado para o aratu e o mesmo fica "paradinho" tornando a coleta mais fácil e rápida. Para auxiliar na captura as marisqueiras utilizam uma vara com linha de fabricação própria. Em uma das extremidades da linha é colocado uma isca (normalmente pequenos crustáceos) para atrair o aratu. Quando a isca é fisgada elas puxam os mesmos e os colocam nos baldes ou latas. Após a volta para o porto as catadoras lavam os aratus e os levam para a casa onde realizam o beneficiamento artesanal. Chegando em casa elas relatam que a depender da hora só fazem o beneficiamento no dia seguinte, já outras relatam que independentemente do horário o beneficiamento é realizado.

Poucos estudos sobre a estrutura populacional desses estoques têm sido realizados. Esse trabalho teve como objetivo principal caracterizar a estrutura populacional e aspectos reprodutivos do G. Cruentata que ocorrem no estuário dos rios Real e Piauí, localizados no sul do estado de Sergipe.

\section{Material e Métodos}

O estudo foi realizado em duas áreas de manguezal, no estuário dos rios Real e Piauí, nas comunidades de Cajazeiras e Pontal. A comunidade de Cajazeiras está localizada nas coordenadas geográficas de $11^{\circ} 25^{\prime} 40,14^{\prime \prime}$ S e $37^{\circ} 26^{\prime} 38,75^{\prime}$ O, faz parte do município de Santa Luzia do Itanhy. A comunidade do Pontal localizada nas coordenadas $11^{\circ} 48^{\prime} 09,19^{\prime}$ 'S e $37^{\circ} 40^{\prime} 21,01^{\prime}$ O , e faz parte do município de Indiaroba (Figura 1).

As coletas foram efetuadas mensalmente sempre na lua nova, no período de março de 2015 a fevereiro de 2016. A coleta foi realizada por pescadoras profissionais, que utilizaram o apetrecho composto por uma

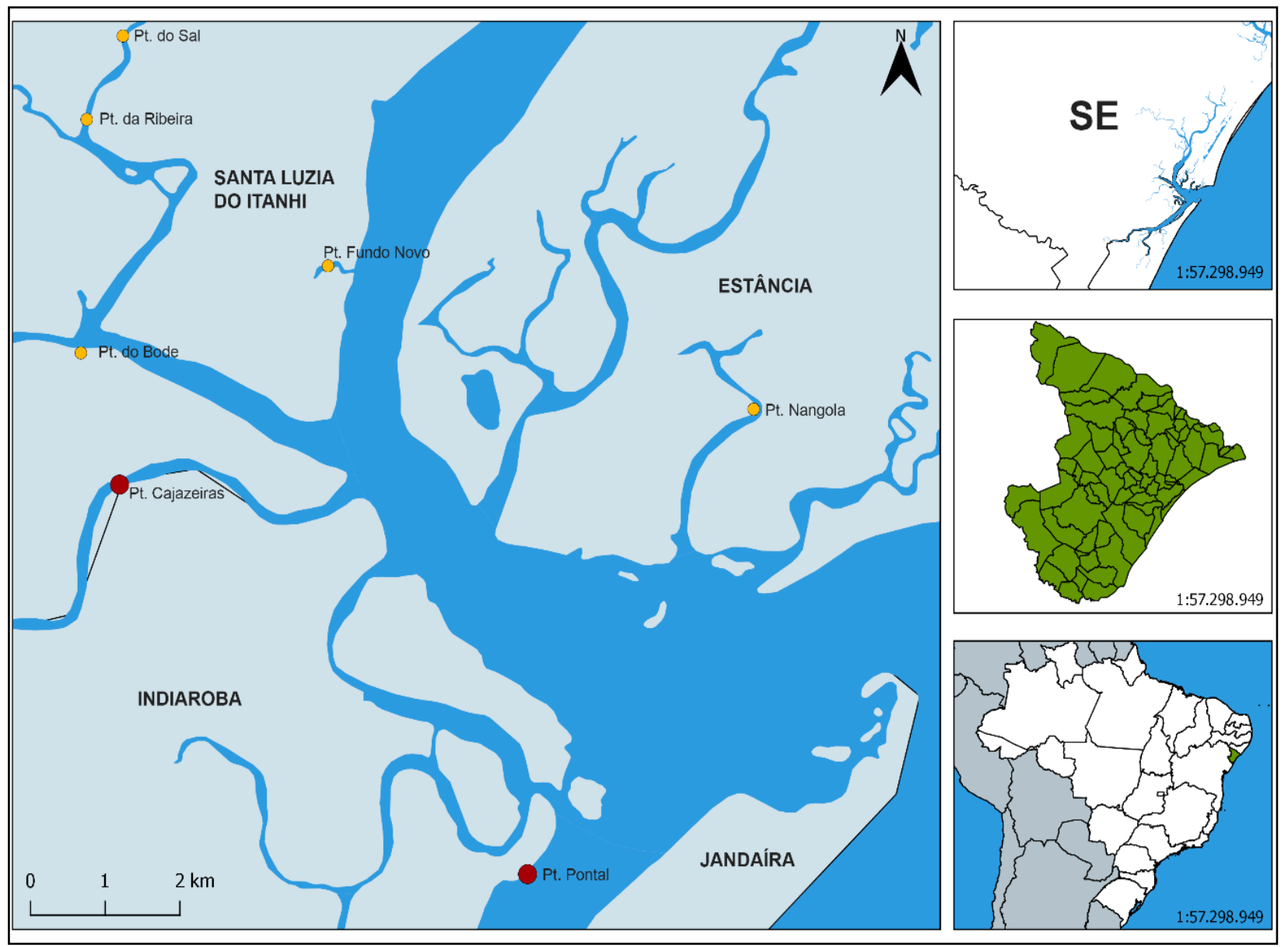

Figura 1. Estuário dos rios Real e Piauí (Adaptado de Cunha, 2015).

vara com linha de nylon em cuja extremidade era fixada uma isca de origem animal. As capturas foram realizadas pelas mesmas pescadoras, padronizado o esforço de pesca de aproximadamente seis horas de trabalho diário, por cada pescadora.

Após a captura os exemplares foram transportados em caixa de isopor com gelo para o laboratório onde foram separados por localidade e se estavam completos (com todas as estruturas) ou incompletos (com 
perdas de estruturas). Posteriormente, cada indivíduo foi sexado através da observação do abdômen (estreito para machos e largo para fêmeas) e foram determinados a largura do cefalotórax (LC), comprimento do cefalotórax (CC) e o peso total (PT). As medidas foram efetuadas com o auxílio de um paquímetro digital $(0,1 \mathrm{~mm})$ e o peso utilizando uma balança digital eletrônica $(0,1 \mathrm{~g})$. Somente os indivíduos considerados completos foram pesados.

As análises macroscópicas das gônadas femininas foram realizadas para determinação dos estágios gonadais e desenvolvimento, os quais foram utilizados a anatomia do apêndice do abdômen e registrando a ocorrência de fêmeas portadoras de ovos aderidos aos pleópodos (fêmea tufada ou ovada). Considerou-se, portanto, dois estágios de desenvolvimento reprodutivo para as fêmeas: Imaturo - fêmea com ovos aderidos; Maduro - sem ovos aderidos.

$\mathrm{Na}$ caracterização de Maduros foi utilizada a descrição proposta por Souza (2006) usando como indicadores o volume e a coloração das gônadas. Para a identificação da coloração das gônadas, o cefalotórax foi removido de forma que foi possível observar o aparelho reprodutor e identificar os estágios gonadais: Estágio I - Pré-vitelogênese - Branco ou amarelo claro sem a presença de ovos; Estágio II Vitelogênese inicial - Laranja com ou sem a presença de ovos; Estágio III - Vitelogênese avançada ou madura - Marrom escuro com ou sem a presença de ovos e Estágio IV- Desovado - Branco ou amarelo claro flácidos sem ovos.

Para os machos a identificação da coloração das gônadas, através da remoção do cefalotórax, identificou-se os estágios de desenvolvimento tais como para as fêmeas: Estágio I - Pré-vitelogênese, Estágio II - Vitelogênese inicial, Estágio III - Vitelogênese avançada ou madura e Estágio IV- Desovado. Considerando os estágios de desenvolvimento reprodutivos classificamos: Imaturos - Estágio I e Maduro os outros estágios.

Para estimar a largura média da primeira maturidade gonadal $\left(\mathrm{L}_{50}\right)$ foi estimada a partir da distribuição da frequência acumulada das fêmeas e machos maduros plotadas contra o valor da largura do cefalotórax, cuja mediana fornece uma estimativa do tamanho em que 50\% dos indivíduos estão aptos a participarem do processo reprodutivo (Ivo \& Fonteles-Filho, 1997). Na estimativa do $\mathrm{L}_{50}$ morfológico e gonadal, utilizou-se a equação da curva logística do programa Statistica, versão 6.0 (Statsoft, 2001). Para verificar possíveis diferenças nas médias de largura da carapaça entre as fêmeas e entre os machos das diferentes áreas de manguezal, foi utilizado o Teste $t$ de Student, $\alpha=0,05$.

\section{Resultados}

Foram estudados 3.291 indivíduos. 53\% foram procedentes dos manguezais do Povoado Cajazeiras e $47 \%$ do manguezal do Povoado Pontal. Os indivíduos procedentes de Cajazeiras totalizaram 896 machos e 843 fêmeas e do Pontal 798 machos e 754 fêmeas (Tabela 1).

A estrutura em comprimento e peso mostrou que a largura de carapaça (LC) dos indivíduos variou em Cajazeiras de 25 a $53,8 \mathrm{~mm}$ e no Pontal de 25 a $59,6 \mathrm{~mm}$. O resultado do teste t para largura de carapaça (LC) e peso total $(\mathrm{PT})$ (valor crítico $=1,96$, para $\alpha=0,05$ e $\mathrm{GL}=1400$ ), foi significante indicando que machos e fêmeas de aratu diferem estatisticamente em largura de carapaça e peso, sendo os machos maiores e mais pesados do que as fêmeas, nas duas áreas (Tabela 1).

Tabela 1. Medidas de tendência central e dispersão para machos e fêmeas de aratu, Goniopsis cruentata, e teste "t" de Student para comparação em largura de carapaça e peso de machos e fêmeas. Indivíduos capturados nas áreas de manguezais dos Povoados de Cajazeiras e Pontal, Sergipe, no período de 2015 e 2016.

\begin{tabular}{lcccccccc}
\hline & \multicolumn{4}{c}{ Cajazeiras } \\
\cline { 2 - 10 } Variáveis & \multicolumn{2}{c}{ LC $(\mathrm{mm})$} & \multicolumn{2}{c}{ PT $(\mathrm{g})$} & \multicolumn{2}{c}{ LC $(\mathrm{mm})$} & \multicolumn{2}{c}{ PT $(\mathrm{g})$} \\
\cline { 2 - 10 } & $\mathrm{F}$ & $\mathrm{M}$ & $\mathrm{F}$ & $\mathrm{M}$ & $\mathrm{F}$ & $\mathrm{M}$ & $\mathrm{F}$ & $\mathrm{M}$ \\
\hline n amostral & 843 & 896 & 286 & 312 & 754 & 798 & 218 & 228 \\
Min - mm & 25,0 & 25,7 & 10,3 & 11,6 & 27,0 & 25,0 & 11,0 & 9,2 \\
Máx -mm & 47,8 & 53,8 & 50,7 & 78,4 & 47,8 & 59,6 & 47,4 & 65,6 \\
Média & 37,3 & 38,9 & 25,7 & 33,4 & 36,9 & 38,9 & 25,2 & 32,4 \\
Varância & 9,2 & 16,9 & 40,3 & 124,2 & 10,6 & 23,4 & 43,1 & 142,6 \\
Teste t & \multicolumn{3}{c}{$-9,38$} & $-10,52$ & $-9,59$ & & $-8,0$ \\
\hline
\end{tabular}


A relação peso-comprimento foi determinada para fêmeas e machos e os coeficientes angulares das equações estimadas para as duas áreas, Cajazeiras e Pontal, foram comparados por meio do teste $\mathrm{t}$ com $\alpha=$ 0,05 e tcrít. $=1,64$, respectivamente, onde obteve-se que os mesmos diferem estatisticamente entre si. Os coeficientes angulares estimados crescem em taxas diferenciadas, quando se relaciona peso e comprimento (Figura 2).

Os coeficientes angulares das fêmeas indicaram que o aratu apresenta alometria negativa, pois o valor de $\mathrm{b}<3$, ou seja, pode-se inferir que o comprimento nas fêmeas cresce a uma taxa relativamente maior que o peso. As duas equações para os machos apresentaram alometria positiva, indicando que crescem a uma taxa relativamente menor que o peso (Figura 2). A partir dos valores estimados para o coeficiente angular das regressões para fêmeas e machos capturados em Cajazeiras e Pontal, o teste $t$ indicou existir diferenças significantes entre os mesmos (tcal. $=-5,31$; tcrit. $=1,71$, e p<0,05) e (tcal. $=-3,07$; tcrit. $=1,71$, e p $<0,05)$, respectivamente.
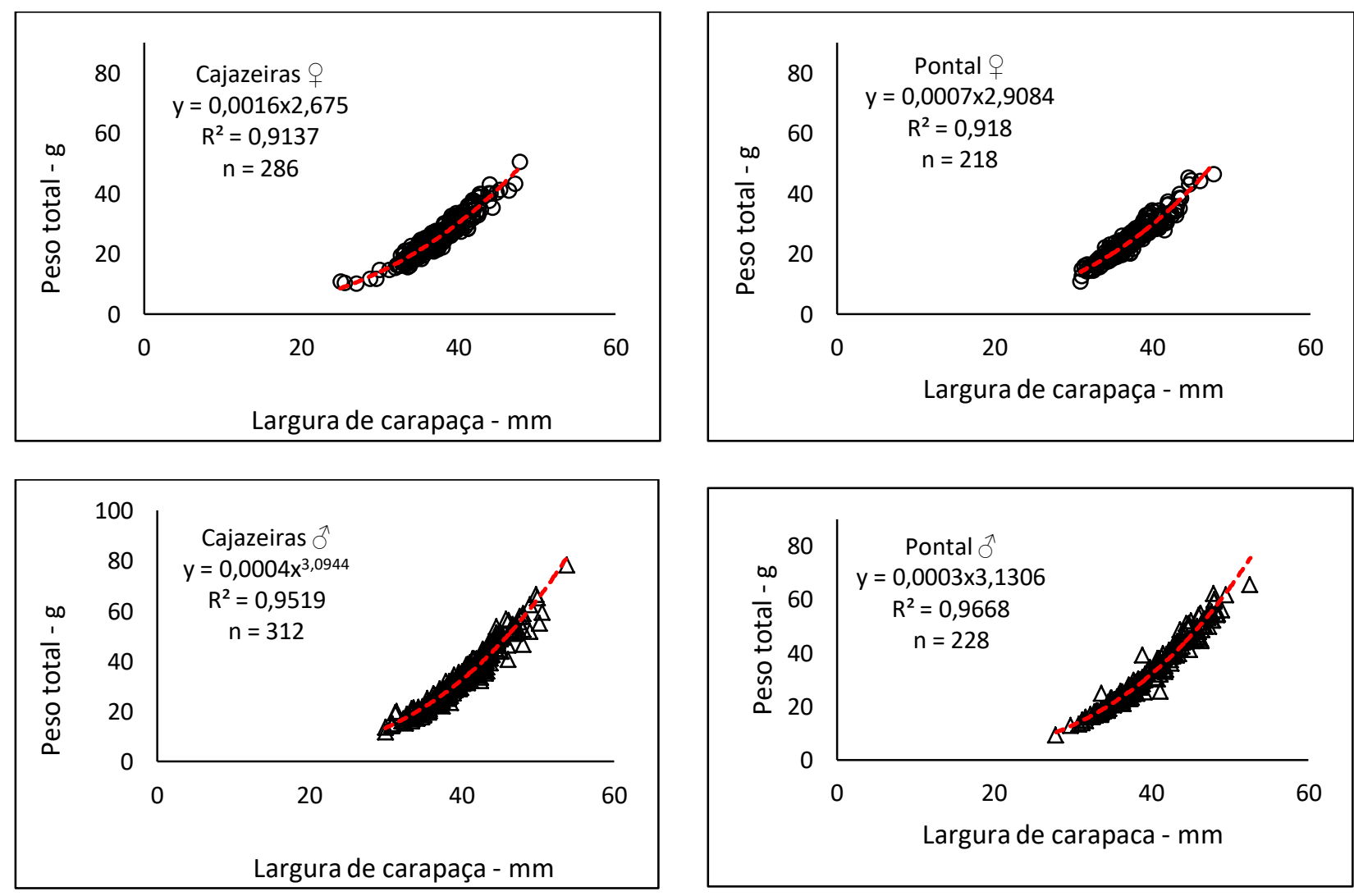

Figura 2. Relação peso/comprimento estimada para machos (a) e fêmeas (b) do aratu, Goniopsis cruentata, indivíduos capturados nas áreas de manguezais dos Povoados de Cajazeiras e Pontal, Sergipe, no período de 2015 e 2016.

Durante todo o período do estudo a proporção sexual geral não diferiu estatisticamente da proporção de1:1 ( $X^{2}$ crit. > 3,84; $X^{2}$ calc. $=1,55$ para Cajazeiras e $X^{2}$ crit. $>3,84 ; X^{2}$ calc. $=1,19$ para o Pontal).

As fêmeas foram maioria nos meses de março, abril, agosto, setembro, novembro e dezembro em Cajazeiras. Em Pontal nos meses janeiro, fevereiro e outubro (Figura 3).

Considerando a frequência de largura de carapaça a proporção sexual entre machos e fêmeas em Cajazeiras e Pontal apresentaram padrões diferentes. As fêmeas foram predominantes nas classes de 26 a 48 $\mathrm{mm}$ em Cajazeiras e no Pontal de 28 a $44 \mathrm{~mm}$. Os machos foram maioria nos maiores comprimentos em Cajazeiras e Pontal, o resultado indica que os machos dominam nos maiores comprimentos (Figura 4).

A menor fêmea ovígera obsevada foi de $25,0 \mathrm{~mm}$ e a maior $47,8 \mathrm{~mm}$ de largura de carapaça, sendo a média de $39,3 \pm 4,1 \mathrm{~mm}$, em Cajazeiras. No pontal a menor foi de 28,8 e a maior $47,4 \mathrm{~mm}$, com média de $36,9 \pm 3,3 \mathrm{~mm}$. Fêmeas ovígeras ocorreram ao longo de todo o período estudado, apresentando menor abundância entre março a junho, nas duas áreas (Figura 5).

A maior frequência de fêmeas encontrada foi de maduras (60\% - Cajazeiras e 57\% - Pontal), seguido por indivíduos em vitelogênese inicial (20\% - Cajazeiras e 19\% - Pontal) e maduros (26\% e 27\% 
respectivamente). Indivíduos na pré-vitelogênese e esvaziados foram 14\% em Cajazeiras e 13\% em Pontal; e $6 \%-11 \%$ respectivamente. A maior quantidade de fêmeas maduros foi encontrada no mês de setembro, em ambas as áreas (Figura 5).
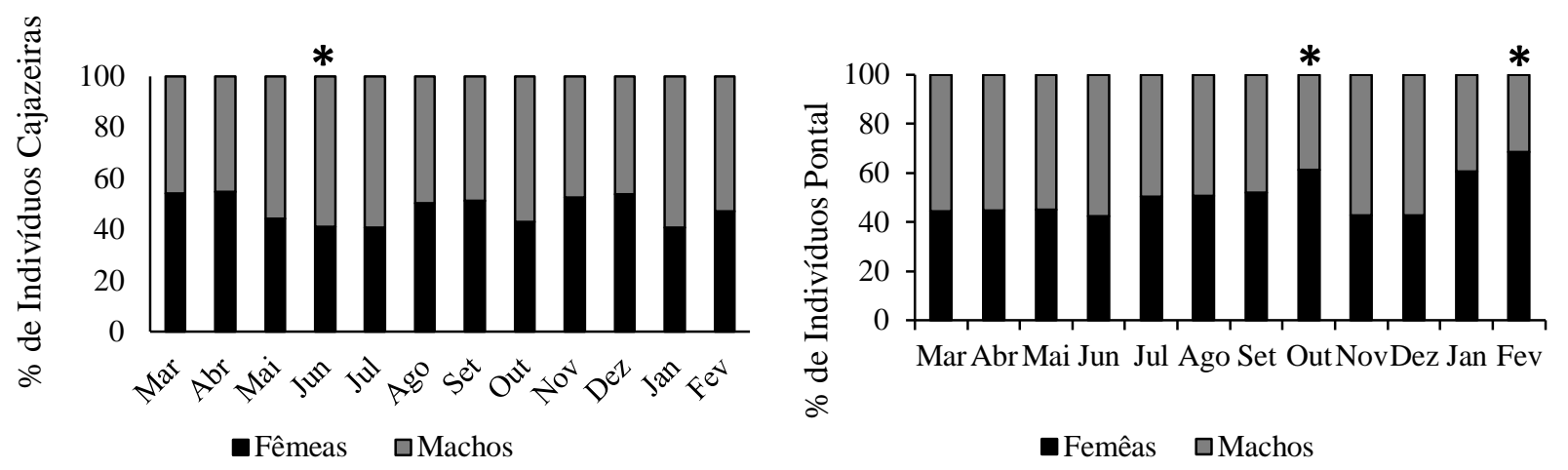

Figura 3. Variação mensal da proporção sexual do aratu, Goniopsis cruentata, capturado no manguezal do povoado Cajazeiras e Pontal, Sergipe, no período de 2015 a 2016.
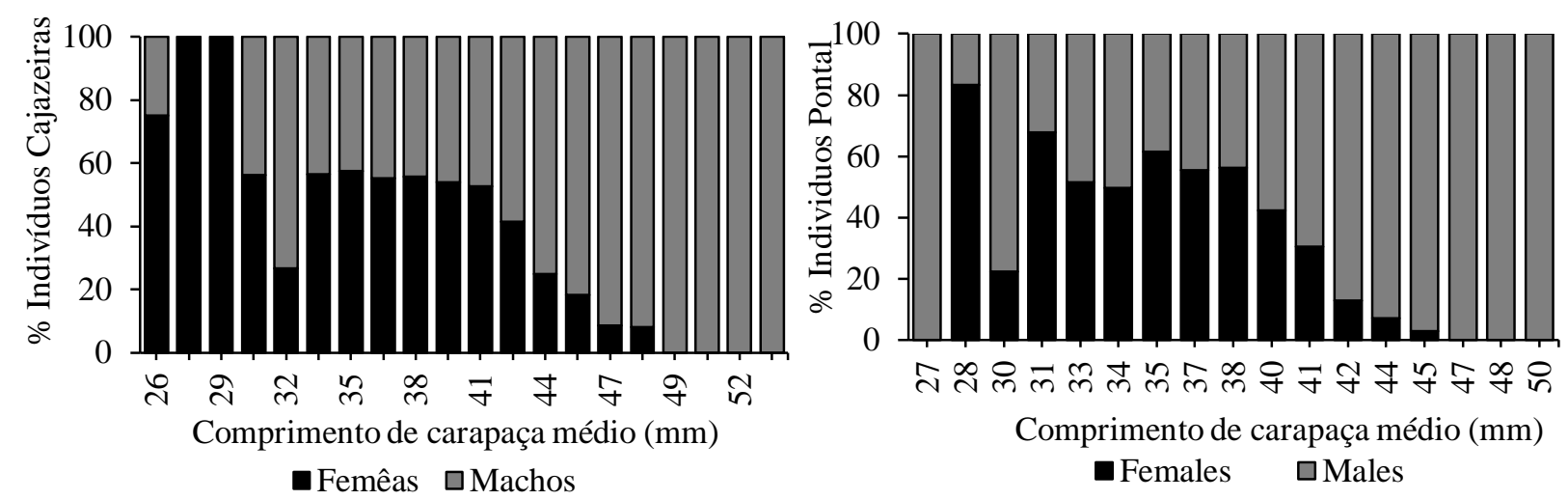

Figura 4. Variação por frequência de largura de carapaça a proporção sexual do aratu, Goniopsis cruentata, capturado no manguezal do povoado Cajazeiras, Sergipe, no período de 2015 a 2016.

A maior frequência de machos encontrada foi de vitelogênese inicial em Cajazeiras (37\%) e prévitelogênese no Pontal (37\%), seguido por indivíduos em pré-vitelogênese e vitelogênese inicial (26\% Cajazeiras e $31 \%$ - Pontal). Machos maduros foram 23\% em Cajazeiras e 20\% em Pontal. Indivíduos esvaziados foram 14\% em Cajazeiras e $12 \%$ em Pontal. A maior quantidade de machos maduros foi encontrada nos meses de maio, setembro e janeiro em Cajazeiras, e junho, setembro e dezembro no Pontal (Figura 5).

Os dados mostraram um aumento na proporção de fêmeas ovígeras nos meses de julho a fevereiro, para as duas áreas estudadas. Em todos os meses foram observados machos maduros (Figura 5). A Figura 6 destaca a frequência relativa de fêmeas ovígeras indicando um pico em setembro nas duas áreas estudadas, evidenciando a ocorrência de um pico reprodutivo.

A frequência relativa de machos maduros foi observada também durante todo o período do estudo com picos em março, junho, setembro e dezembro para o Pontal. Para Cajazeiras foram observados picos em janeiro, junho e setembro. 
Cajazeiras ㅇ

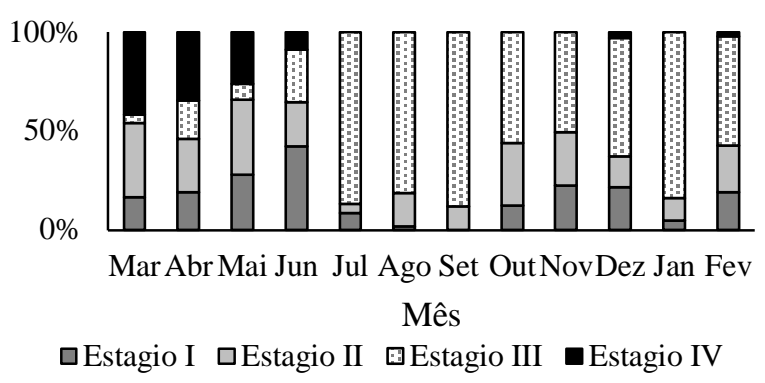

Cajazeiras $\widehat{O}$

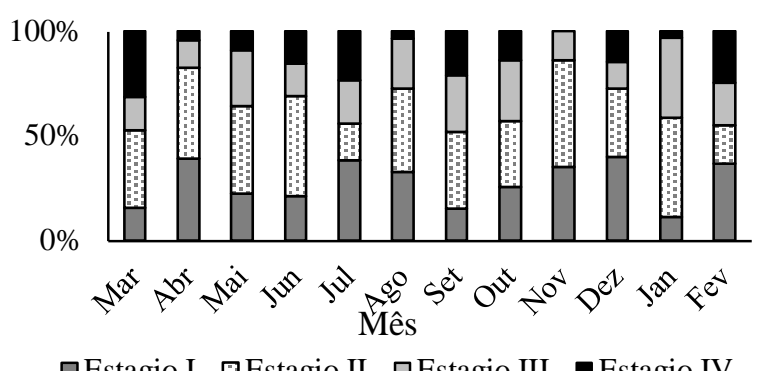

Pontal 우

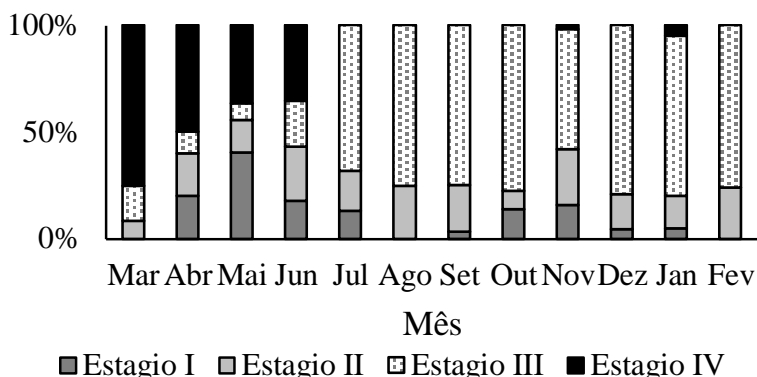

Pontal $\overbrace{}^{\lambda}$

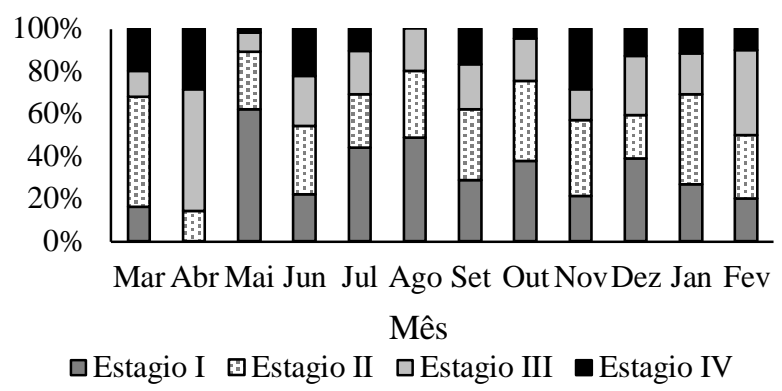

Figura 5. Variação mensal na proporção dos estádios de maturação de fêmeas e machos de Goniopsis cruentata em Sergipe entre março de 2015 e fevereiro de 2016 (Estágio I-Pré-vitelogênese, Estágio II-vitelogênese inicial, Estágio III-vitelogênese avançada ou madura e Estágio IV-desovado).

O resultado estimado da largura de carapaça média na primeira maturação de fêmeas indicou valor de $33,2 \mathrm{~mm}$ e para os machos foi de 36,5 mm para os indivíduos capturas em Cajazeiras (Figura 7). Para os indivíduos capturadas em Pontal o resultado estimado da largura de carapaça média na primeira maturação de fêmeas indicou valor de 31,2 mm e para machos, 36,8 $\mathrm{mm}$ (Figura 8).

O resultado mostrou ainda que $31 \%$ dos machos e $7 \%$ das fêmeas capturadas de Cajazeiras e $36 \%$ dos machos e $3 \%$ das fêmeas capturados em Pontal estão abaixo do tamanho de primeira maturação sexual.
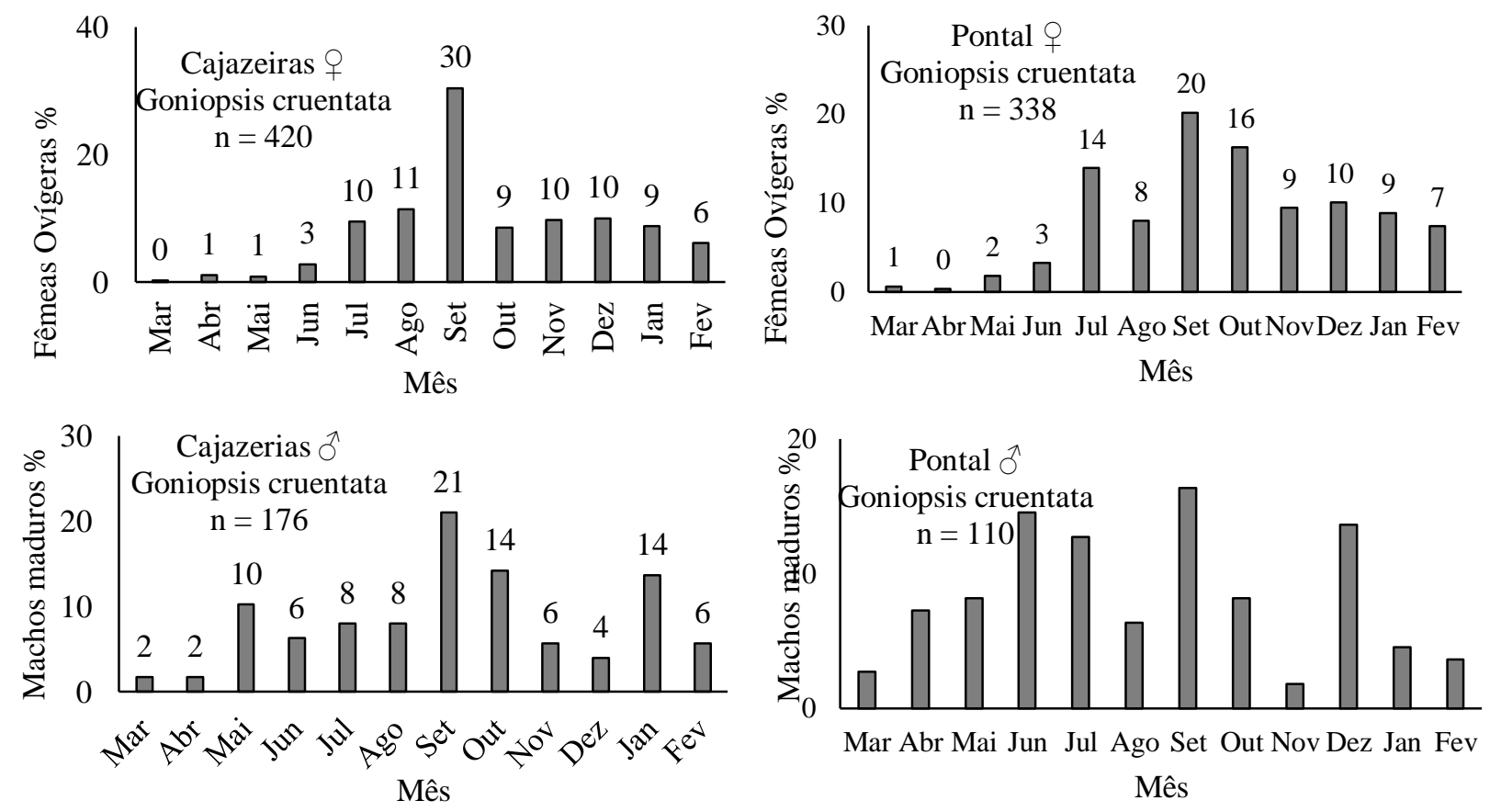

Figura 6. Frequência absoluta mensal de fêmeas ovígeras e machos maduros da espécie Goniopsis cruentata evidenciando o período de picos reprodutivos, capturado no manguezal do povoado Cajazeiras, Sergipe, no período de 2015 a 2016. 

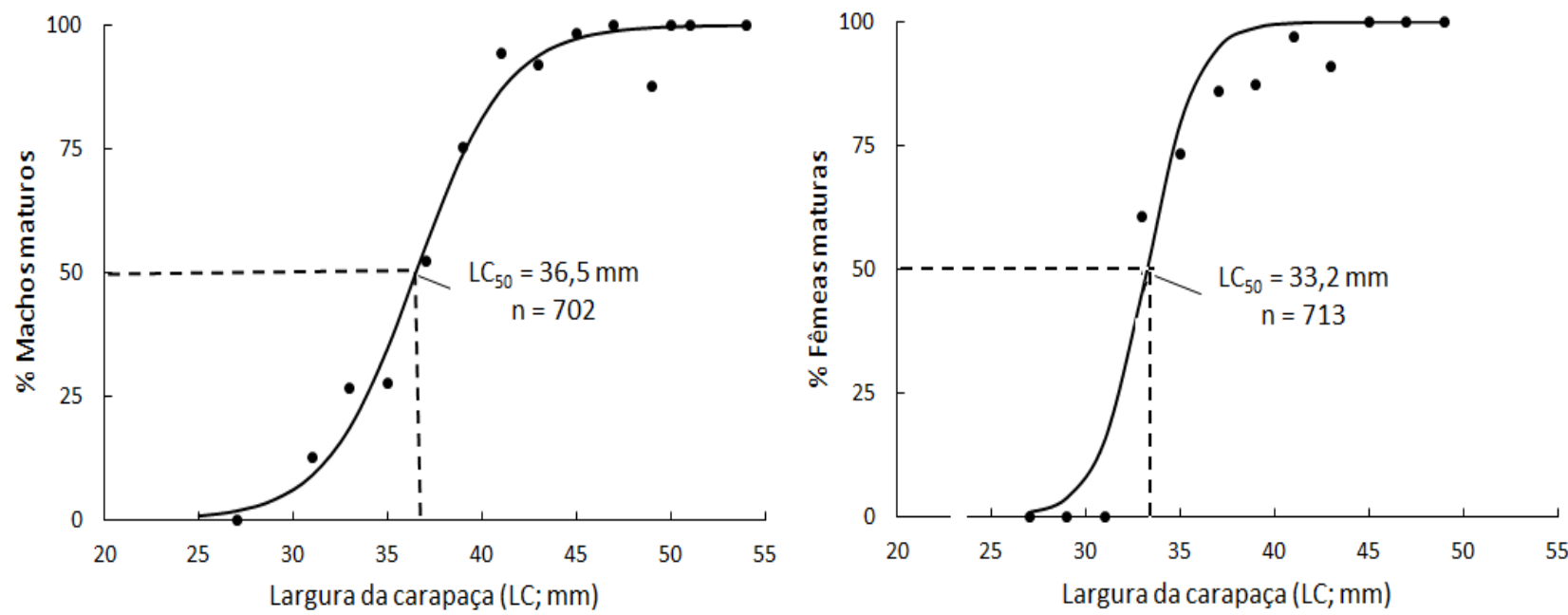

Figura 7. Distribuição de frequência da largura do cefalotórax de fêmeas e machos de aratu com estimativa do tamanho, em que 50\% são considerados fisiologicamente maduros para o estuário do Cajazeiras em 2015/ 2016.
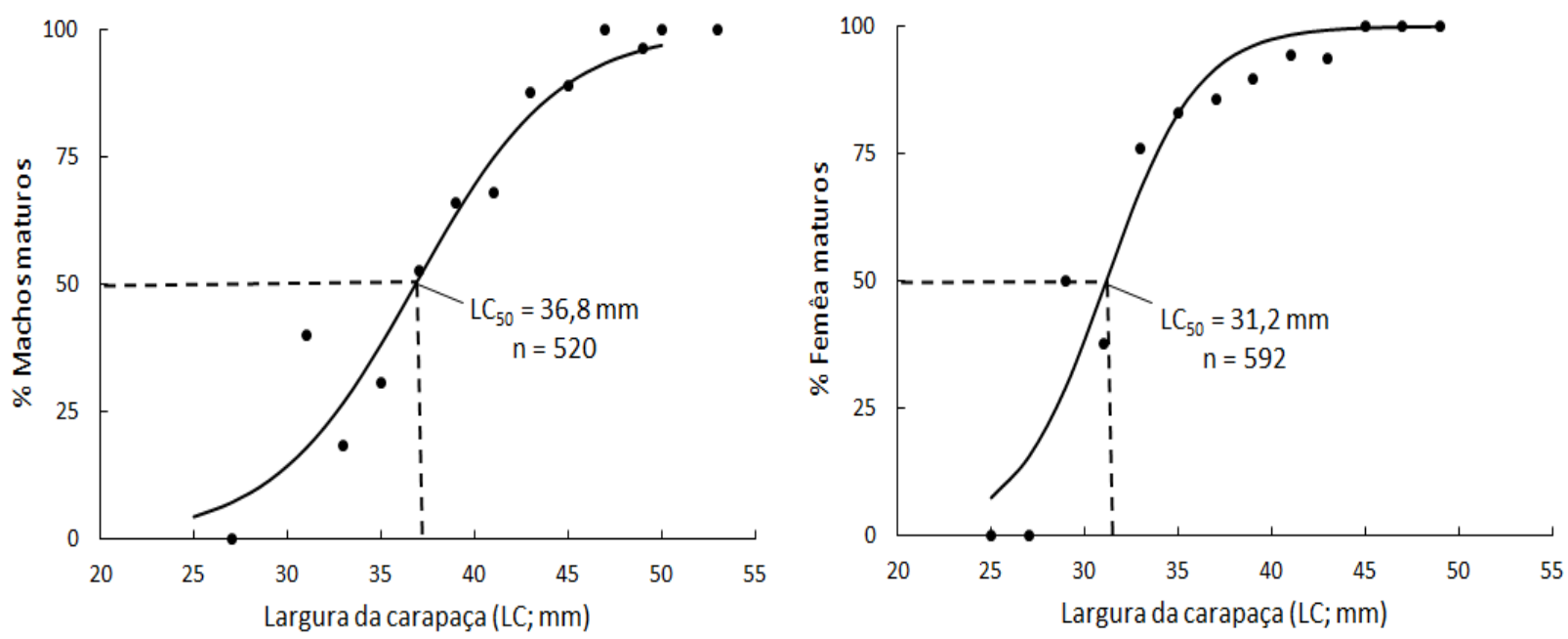

Figura 8. Distribuição de frequência da largura do cefalotórax de fêmeas e machos de aratu com estimativa do tamanho, em que 50\% são considerados fisiologicamente maduros para o estuário do Pontal, em 2015/ 2016.

\section{Discussão}

O estudo revelou que nas duas comunidades a pesca do aratu é intensa e praticada por mulheres como geração de trabalho e renda. A comercialização do aratu é realizada de forma individual, pelas próprias pescadoras ou pelo atravessador. A carne de aratu faz parte da culinária local com alta demanda tanto pela carne processada artesanalmente, como pelo indivíduo inteiro.

Neste estudo analisaram-se as flutuações na estrutura populacional e mudanças corporais ao longo do ano. Analisou duas áreas no estuário do rio Piaui/Real, onde indivíduos foram capturados com a mesma técnica durante o dia. As duas populações diferiram significativamente no tamanho do corpo. Os dados mostraram que a comunidade do Pontal está capturando indivíduos menores, indicando maior esforço de pesca ocasionando diminuição na quantidade de indivíduos maiores (adultos).

O tamanho do corpo é provavelmente a característica quantitativa mais importante de um indivíduo. Afeta praticamente todas as ações fisiológicas de sucesso do indivíduo, tais como as características da fecundidade e acasalamento, produzindo, relações alométricas bem compreendidas dentro e entre organismos (McNab, 1971; Blanckenhorn, \& Demont, 2004).

Flutuações na estrutura populacional geralmente estão associadas naturalmente a mudanças na disponibilidade de alimentos, condições ambientais, ou ataque de um novo predador em qualquer fase do 
ciclo de vida dos indivíduos. E pela ação antrópica em virtude do aumento do esforço de pesca com captura de indivíduos juvenis que resultará em mudanças na taxa de recrutamento juvenil da população. No presente estudo, nas duas áreas, os machos de aratu foram significativamente maiores e mais pesados do que as fêmeas. Os resultados corroboram com os encontrados em outras áreas estuarinas do estado de Sergipe. Hirose et al. (2015) encontrou tamanho médio de comprimento de carapaça para fêmeas de 32,71 e $31,41 \mathrm{~mm}$, e machos 34,12 e 33,02 mm nos manguezais do rio Sergipe e Vaza Barris no estado de Sergipe. Nas áreas próximas ao estado de Sergipe, como por exemplo no complexo estuarino-lagunar Mundaú/Mangaba (Menezes et al., 2012) e manguezais de Caravelas (BA) também foi registrado que machos foram maiores e mais pesados que as fêmeas (Santos et al., 2013). Essa é uma característica dos Brachyura, onde a dinâmica reprodutiva das fêmeas exige maior investimento energético para a produção dos ovos, enquanto os machos investem mais em crescimento, e isso permite que os machos atinjam tamanhos superiores aos das fêmeas em muitas espécies (Hartnoll,1985, Díaz \& Conde, 1989; Cobo, 1995; Araújo et al., 2012).

Nesse estudo as fêmeas foram maioria nos meses de janeiro, fevereiro e de julho a outubro em Pontal. Em Cajazeiras foram maioria em março, abril, agosto, setembro, novembro e dezembro. Estudos realizados no estuário do rio Camaragibe mostraram que fêmeas foram maioria nos meses de maio, outubro e novembro (Botelho et al., 2004).

As equações das relações peso total/comprimento total foram estimadas separadamente para machos e fêmeas do aratu, nas duas áreas, o conhecimento da relação peso-comprimento é importante para descrever os aspectos ligados à biologia e à ecologia das espécies aquáticas, pois ajuda na caracterização dos estoques e permite estabelecer normas para a administração dos recursos pesqueiros (Vazzoler, 1996, Isaac et al., 2004). A partir dos valores estimados para o coeficiente angular das regressões, para fêmeas e machos capturados em Cajazeiras e Pontal, existe diferenças significantes entre os mesmos. A largura de carapaça (comprimento) nas fêmeas cresce a uma taxa relativamente maior que o peso. Os machos apresentaram alometria positiva, indicando que crescem a uma taxa relativamente menor que o peso. Essa diferença pode ser devido ao crescimento relativo da quela, que geralmente é mais acelerado nos machos, por exercer um importante papel na coorte e acasalamento, bem como na defesa do território (Hartnoll, 1985; Leite et al., 2006, Menezes et al., 2012). Essa relação tem mostrado a maneira mais fácil e rápida de descrever o crescimento sem levar em conta a idade, por isso essa estimativa assume importante aplicação na biologia pesqueira (Richter et al., 2000).

O coeficiente angular da relação peso-comprimento é suscetível a variações entre populações diferentes, entre sexos em uma população que habita o mesmo local, durante os meses do ano ou durante as fases de crescimento (Gonzalez \& Ramirez, 1988). Essas variações podem estar relacionadas às diferenças de condições ambientais e a aspectos biogenéticos inerentes a cada espécie (Wenner, 1972).

Durante todo o período do estudo a proporção sexual geral não diferiu estatisticamente da proporção de1:1. Cajazeiras e Pontal apresentaram padrões diferentes para a porcentagem de fêmeas e machos, considerando a frequência de largura de carapaça. Provavelmente a pesca artesanal do aratu tem como alvo indivíduos maiores, pelo fato de que durante o beneficiamento artesanal a quantidade de carne é maior com menor tempo de trabalho e durante a comercialização de indivíduos inteiros a procura é por indivíduos maiores. A seleção de indivíduos maiores pelas pescadoras tenderia a capturar maior número de machos devido ao seu maior tamanho do corpo. Isso poderia explicar o desvio em proporção sexual registrada para os adultos em alguns estudos.

Esse resultado indica existir a mesma tendência de proporção sexual para outras áreas de manguezais do estado de Sergipe como as áreas dos rios Vaza Barris e Sergipe (Hirose et al., 2015). Outros estudos apontam que as fêmeas foram superiores aos machos, considerando a frequência mensal, Cobo (1995) registrou 48\% de machos para o litoral de São Paulo e Moura (1998) 59\% de machos para o litoral norte de Pernambuco. Wenner (1972) e Margalef (1977) afirmaram que é comum a disparidade entre sexos nos crustáceos, cujas causas podem ser devido ao comportamento reprodutivo, fatores ambientais, longevidade, taxa de mortalidade e ação antrópica.

Fêmeas maduras foram encontradas durante todo o período estudado com menor pico no período de menor pluviosidade (mais seco), indicando que a atividade sexual dessa espécie é contínua. Em contraponto, Cobo \& Fransozo (1999) observaram fêmeas com ovos exteriorizados durante todos os meses com exceção do mês de julho. Santos et al. (2001) registraram um pico de fêmeas ovígeras em março e outro em setembro para o litoral sul de Pernambuco. Cobo (1995) registrou para Ubatuba, litoral de São Paulo, dois picos de fêmeas ovígeras, um entre dezembro a abril e outro em setembro. De modo geral é possível afirmar que no litoral brasileiro o Goniopsis cruentata apresenta uma desova no verão e outra entre 
o inverno e a primavera (Santos et al., 2013). O efeito da pesca no potencial reprodutivo de G. cruentata deve ser considerado uma vez que os resultados mostraram que aproximadamente $42 \%$ das fêmeas capturadas em Cajazeiras e 38\% do Pontal carregavam ovos. De acordo com nossa observação, as fêmeas ovígeras são normalmente utilizadas na produção de "catado" em Sergipe, que poderia pesadamente impactar o potencial reprodutivo do estoque.

Cobo (1995) menciona que para a população de Goniopsis cruentata, após o período larval, as fêmeas, como parte de sua estratégia reprodutiva, passam a apresentar um crescimento mais acelerado em relação ao dos machos, com a finalidade de atingir precocemente a maturidade sexual, quando então concentram seus recursos na maturação de gônadas e na produção de ovos. Em Brachyura, o crescimento e a reprodução estão sob controle hormonal, existindo uma certa oposição entre esses hormônios, portanto, ou o animal encontra-se em fase de crescimento ou de reprodução. Desta forma, é comum aos machos atingirem maiores comprimentos em relação às fêmeas (Diaz \& Conde, 1989; Cobo, 1995; Moura, 1998).

O tamanho em início da maturidade sexual (L50\%) é regularmente utilizado para definir um tamanho mínimo de captura. Para o aratu nesse estudo foi estimado entre 33,2 e 31,2 para fêmeas e 36,5 e 36,8 mm para machos. Hirose et al (2015) estimou o tamanho da maturidade s sexual (L50\%) para indivíduos capturados no estuário do ri Sergipe em 32,38 para machos e 26,53 para fêmeas, e para o estuário do rio Vaza-Barris em 30,90 para machos e 27,88 para fêmeas. Os resultados indicam haver maior esforço de pesca nessa área ou maior perda de hábitat.

O resultado mostrou ainda que $31 \%$ dos machos e $7 \%$ das fêmeas capturadas de Cajazeiras e $36 \%$ dos machos e 3\% das fêmeas capturados em Pontal estão abaixo do tamanho de primeira maturação sexual. Esse resultado indica que mais de 50\% dos jovens são mantidos na população e que provavelmente é possível existir um esforço de pesca maior sobre os machos nos dois locais estudados. As populações de aratu ainda se mantem em níveis de explotação, embora tenha sido registrado capturas maiores sobre os machos. Mais estudos devem ser realizados para subsidiar a gestão dessa pescaria em Sergipe.

Os resultados indicaram uma tendência em direção a uma diminuição no tamanho do corpo, abundância e potencial reprodutivo de G. cruentata, provavelmente em resposta à pressão da pesca e perda de habitat. É necessário um plano de manejo para a espécie visando sustentabilidade para esta atividade que promove a subsistência de pescadoras e suas famílias.

\section{Referências}

Araújo, A. R.R.; Barbosa, J.M.; Santos, J. P.; Carvalho, B. L.F.; Filho, E. B.G.; Deda, M. S. \& Silva, C. O. (2016). Boletim estatístico da pesca nos litorais de Sergipe e extremo Norte da Bahia. 6(1):1-100. Editora UFS: Aracaju.

Araújo, M.S.L.C., Castiglioni, D.S. \& Coelho, P.A. (2012). Width-weight relationship and condition factor of Ucides cordatus (Crustacea, Decapoda, Ucididae) at tropical mangroves of Northeast Brazil. Iheringia, Série Zoologia, Porto Alegre, 102(3):277-284.

Blanckenhorn, W.U. \& Demont, M. (2004). Bergmann and converse Bergmann latitudinal clines in arthropods: two ends of a continuum? Integr. Comp. Biol. 44:413-424.

Botelho, E. R. O., Andrade, C. E. R. \& Santos, M. C. F. (2004). Estudo da população de aratu-do-mangue Goniopsis cruentata (Latreille, 1803) (Crustacea, Decapoda, Grapsidae) no estuário do rio Camaragibe (Alagoas - Brasil). Boletim. Técnico-científico do Cepene, Tamandaré, 12(1): 91-98.

Bowman, T.E. \& Abele,L.G. (1982). Classification of the recent crustacea. In: Abele, L.G. (ed) The biology of crustácea, systematics, the fóssil record and biogeography. Academic Press, New York, 1(1): 1-25.

Cobo, V.J. \& A. Fransozo, A. (1998). Relative growth of Goniopsis cruentata (Crustacea, Brachyura, Grapsidae), on the Ubatuba region, São Paulo, Brazil. Iheringia, Série Zoologia, 84: 21-28.

Cobo, V.J. \&. Fransozo. (1999). Fecundity and reprodution period of the red mangrove crab Goniopsis cruentata (Brachyura, Grapsidae) São Paulo state, Brazil, p. 527-533. In: J.C. Vaupel Von Klein \& F.R. Schram (Eds). The Biodiversity crisis and Crustacea. Rotterdam, A.A. Balkema Publishers, Crustacean Issues, Vol. 12:848.

Cobo, V. J. (1995). Biologia populacional e crescimento relativo de Goniopsis cruentata (Latreille, 1803) (Crustácea, Decapoda, Grapsidae), na região de Ubatuba, SP. 1995, 79f. Dissertação (Mestrado em Zoologia) - Unesp, Botucatu. 
Coelho, P. A. (1965). Os crustáceos decápodos de alguns manguezais pernambucanos. Trabalhos do Instituto Oceanográfico da Universidade Federal de Pernambuco, 7: 71-90.

Fransozo, A., Mantelatto, F.L.M., Bertini, G., Fernandes-Góes, L.C. \& Martinelli, J.M. (1998). Distribution and assemblages of anomuran crustaceans in Ubatuba Bay, north coast of São Paulo State, Brazil. Acta Biol. Venez. 18(4): 17-25.

Diaz, H. \& Conde, J. E. (1989). Population dynamics and life history of the mangrove crab Aratus pisonii (Brachyura, Grapsidae) in a marine environment. Bull. Mar. Sci., Miami, 45(1): 148-163.

Gonzalez, S. A. \& I. Ramirez, M. F. (1988). Biologia de la sardina de rio, Astyanax bimaculatus (Linnaeus, 1758) (Pisces: Characidae) del rio Cancamure, Estado Sucre, Venezuela. 3. Biometria. Boletin del Instituto Oceanografico de Venezuela, 27(2):149-155.

Haley, S.R. (1979). Sex ration as a fuction of size in Hippa pacifica Dana (Crustacea, Anomura, Hippidae): a test of sex reversal and differential growth rate hypotheses. Americam Naturalist, 113(3): 391-397.

Hartnoll, R. G. (1985). Growth, sexual maturity and reproductive output. In: Werner, A.M. (ed.), Factors in adult growth. A. A. Balkema Publishers, Rotterdam, 3: 101-128.

Hirose, G.L., Souza, L.S.; Silva, S.L.R.; Alves, D.F.R. \& Negreiros-Fransozo, M.L. (2015) Population structure of the red mangrove crab, Goniopsis cruentata (Decapoda: Grapsidae) under different fishery impacts: Implications for resource management. Rev. Biol. Trop. 63(2): 443-457.

Isaac, V. J.; Silva. C. O. \& Ruffino M. L. (2004). A pesca no Baixo Amazonas. In: Ruffino. M. L. A pesca e os recursos pesqueiros na Amazônia brasileira. Manaus: Ibama/Provárzea. p. 185-212.

Ivo, C.T.C. \& Fonteles-Filho, A.A. 1997 Estatística pesqueira: Aplicação em Engenharia de Pesca. Fortaleza: Tom Gráfica e Editora.

Leite, M. M. L. \& Fonteles-Filho, A. A.; Silva, J. R. F.; Cardoso, N. S. (2006). Análise do crescimento alométrico no caranguejo-uçá, Ucides cordatus (Decapoda:Ocypodidae), no estuário do Rio Coreaú, Camocim, Ceará. Arquivos de Ciências do Mar, 39: 93-98.

Leme, M. H. A. (1999). Estratégia reprodutiva de duas espécies de Grapsídeos (Crustácea,Brachyura, Sesarminae) de manguezais. Universidade Estadual Paulista Botucatu, São Paulo. Tese de doutorado.

Maciel, D. C. \& Alves, A. G. C. (2009). Conhecimentos e práticas locais relacionados ao aratu Goniopsis cruentata (Latreille, 1803) em Barra de Sirinhaém, litoral sul de Pernambuco. Biota Neotrop., 9(4).

McNab, B.K. (1971) On the ecological significance of Bergmann's rule. Ecology, 52:845-854.

Margalef, R. (1977). Ecologia 2. ed. Barcelona: Omega.

Melo, G.A.S. (1996). Manual de identificação dos Brachyura (caranguejos e siris) do litoral brasileiro. São Paulo: Museu de Zoologia da Universidade de São Paulo/Editora Plêiade/Fapesp.

Menezes, A. P. D., Araújo, M. S. L. C., \& Calado, T.C. S. (2012). Bioecologia de Goniopsis cruentata (Latreille, 1803) (Decapoda, Grapsidae) do complex estuarino-lagunar Mundaú/Manguaba, Alagoas, Brasil.Natural Resources, 2(2), 37-49.

Moura, N. F. O. (1998). Aspectos bioecológicos do aratu Goniopsis cruentata (Latreille, 1803) no estuário do rio Paripe - Itamaracá - PE. 79p. Monografia (Bacharelado em Ciências Biológicas) - Universidade Federal de Pernambuco, Recife.

Moura, N. F. O. \& Coelho, P. A. (2004). Maturidade sexual fisiológica em Goniopsis cruentata (Latreille) (Crustacea, Brachyura, Grapsidae) no estuário do Paripe, Pernambuco, Brasil. Rev. Bras. Zool., Curitiba, 21(4): 1011-1015.

Oshiro, L.M.O.: R. Silva \& Silva, Z. S. (1998). Compos ição da fauna de braquiúros (Crustacea. Decapoda) dos Manguezais da Baía de Sepetiba - RJ . Nauplius, 6(3): 1-40.

Pianka, E. R. (1974). Evolutionary ecology. New York, Harper \& Row Publishers.

Poole, R.W. (1974). Introduction to quantitative ecology. Tokyo: Mc Graw-Hill. 
Richter, H. C., Luckstadt, C., Focker, U. \& Becker, K. (2000). An improved procedure to assess fish condition on the basis of length-weight relationships. Archive of Fishery and Marine Research, 48(3): 255264.

Ricklefs, R. E. (2010). A economia da natureza. In A economia da natureza. Guanabara Koogan.

Ricklefs, R.E. (2004). A comprehensive framework for global patterns in biodiversity. Ecol. Lett. 7: 1-15. doi:10.1046/j.1461-0248.2003.00554.x

Santos, M. C. F.; Botelho, E. R. O. \& Ivo, C. T. C. (2001). Biologia populacional e manejo da pesca de aratu, Goniopsis cruentata (Latreille 1803) (Crustacea: Decapoda: Grapsidae) no litoral sul de Pernambuco - Brasil. Boletim Técnico-científico do Cepene, 9: 87-123.

Santos, M. C. F.; Botelho, E. R. O. \& Ivo, C. T. C. (2001). Biologia populacional e manejo da pesca de aratu, Goniopsis cruentata (Latreille 1803) (Crustacea: Decapoda: Grapsidae) no litoral sul de Pernambuco - Brasil. Boletim Técnico-científico do Cepene, 9: 87-123.

Santos. M.C.F.; Botelho..E.R.O; Corrêa..F.M.; Almeida.L.; Silva.C.G.M. (2013). Aspectos populacionais do aratu Goniopsis cruentata (Latreille, 1803) (Decapoda, Grapsidae) no manguezal de Caravelas (Bahia Brasil). Boletim Técnico-científico do Cepene, 19(1): 27-36.

Sousa, E. C. \& Calado, T. C. S. (2000). A família Grapsidae Macleay, 1838 (Crustacea: Decapoda: Brachyura) no Complexo Estuarino-lagunar Mundaú/Manguaba -Alagoas. Boletim Nível Estudos Ciências Marinhas, 11: 119-141.

Souza, L.P. (2008). Maturidade sexual e relações morfométricas do caranguejo Goniopsis cruentata (Latreille, 1803), (Crustacea: Brachyura:Grapsidae) do estuario do Rio Jaguaribe (Aracati-Ceará). [Dissertacao de Mestrado]. Fortaleza (CE): Universidade Federal do Ceará.

Statsoft (2001). Statistica for Windows. Computer program manual. Tulsa: Statsoft, Inc., 2001.

Sternberg, R. V. (1994). Systematic implications of color pattern polymorphism in Goniopsis pulchra (Decapoda: Brachyura: Grapsidae) from Ecuador. Proceedings of the Biology Society of Washington, 107(4): 721-728.

Vazzoler, A. D. M. (1996). Biologia da reprodução de peixes teleósteos: teoria e prática. Maringá, Universidade Estadual de Maringá: Maringá.

Wenner, A. M. (1972). Sex-ratio as a function of size in marine Crustacea. Amer. Nat., Chicago, 106(949): 321-350. 